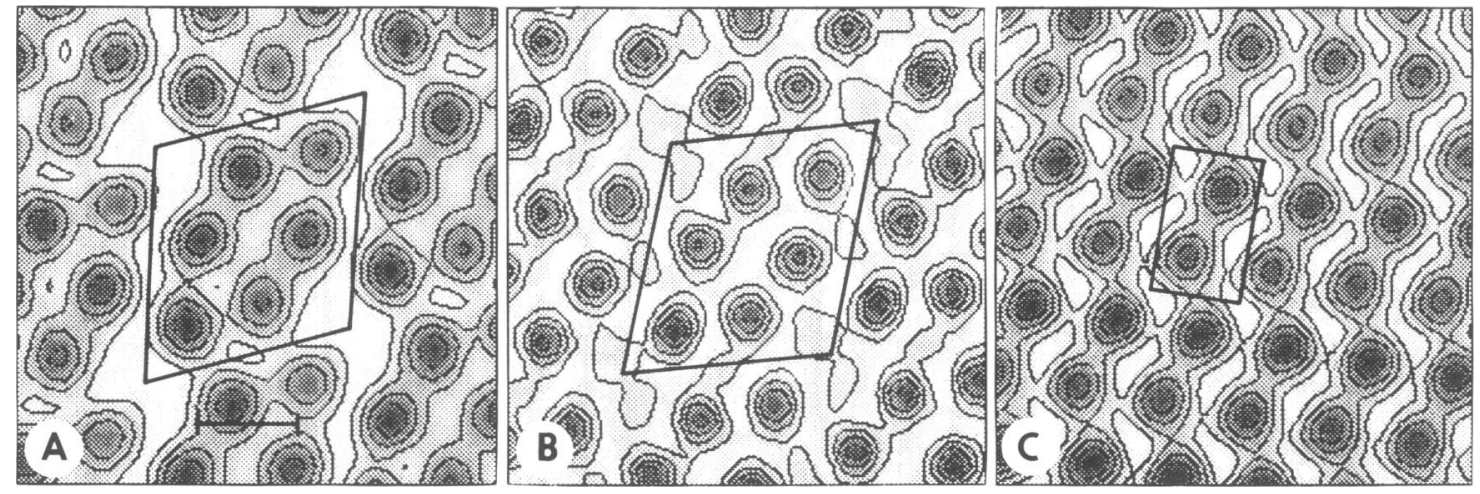

FigurE 1 Computer filtered images of outer mitochondrial membranes containing oblique $(A, B)$ and rectangular $(C)$ lattices, stained with potassium phosphotungstate $(A, C)$ or uranyl acetate $(B)$ as described in footnote of Table I. Micrographs taken with a Philips 301 (Philips Electronic Instruments, Inc., Mahwah, NJ) operated at $100 \mathrm{kV}(A, B)$ or a JEOL 200 at $150 \mathrm{kV}(C)$ (JEOL USA, Electron Optics Div., Cranford, NJ). Each image was reconstructed by inverse transformation of the discrete Fourier spectrum from a single membrane layer. The scale in each image is the same; the bar in $A$ represents $5 \mathrm{~nm}$. Contour lines and unit cells are superimposed.

to negative-stain specimen preparation (details of which are presented in footnote to Table I). When $0.1 \mathrm{mM}$ EDTA is included in the dialysis buffer, the lattice parameters are unaffected (Table I, specimen 2) although there are indications in the filtered images of decreased phosphotungstate accumulation by the subunits (data not shown). On the other hand, when the dialysis medium contains $0.1 \mathrm{mM} \mathrm{CaCl}$, the predominant lattice is rectangular (or near-rectangular) with unit cells large enough to accommodate only two stain-accumulating sites (Table I, specimen 4). With phosphotungstate, the stain centers in the new lattice display the same diameters and nearestneighbor distances, 4.5 to $5 \mathrm{~nm}$, as in the oblique lattice, although their packing is now nearly hexagonal (Fig. $1 C$ ). The change in ordering of the outer mitochondrial membrane subunits induced by calcium pretreatment is interesting in light of evidence that similar treatment alters the accessibility of substrate molecules to enzymes in the intermembrane space of intact plant mitochondria $(6,4)$. The calcium-induced symmetry change may, for example, reflect a permeability-related conformation change in the outer membrane subunits. Alternatively, the lattice changes associated with calcium exposure may be lipid-related, e.g., the result of endogenous phospholipase activation. These two possibilities are currently under investigation.

This material is based upon work supported by grant PCM-8021789 from the National Science Foundation and grant 37005-80 from Health Research, Inc.

Received for publication 29 April 1981.

\section{REFERENCES}

1. Parsons, D. F., W. D. Bonner, Jr., and J. G. Verboon. 1965. Electron microscopy of isolated plant mitochondria and plastids using both the thin-section and negative-staining techniques. Canad. J. Botany. 43:647-655.

2. Colombini, M. 1980. On the structure of a channel-forming protein functionally purified from mitochondria. Fed. Proc. 39:1812.

3. Zalman, L. S., H. Nikaido, and Y. Kagawa. 1980. Mitochondrial outer membrane contains a protein producing nonspecific diffusion channels. J. Biol. Chem. 255:1771-1774.

4. Douce, R., C. A. Mannella, and W. D. Bonner, Jr. 1973. The external NADH dehydrogenases of intact plant mitochondria. Biochim. Biophys. Acta. 292:105-116.

5. Mannella, C. A., and J. Frank. 1980. Lattice structure in the outer membrane of Neurospora mitochondria. In Electron Microscopy 1980. P. Brederoo and W. De Priester, editors. Seventh European Congress on Electron Microscopy Fdn., Leiden, The Netherlands. 2:618-619.

6. Hackett, D. P. 1961. Effects of salts on DPNH oxidase activity and structure of sweet potato mitochondria. Plant Physiol. 36:445-452.

\title{
PHOTOACOUSTIC CALORIMETRY OF PURPLE
}

\section{MEMBRANE}

\author{
Jane LeGrange, David Cahen, and S. Roy Caplan \\ Departments of Membrane Research and Structural Chemistry. The Weizmann Institute of Science, \\ 76100 Rehovot, Israel.
}

Photoacoustic measurements on suspensions of purple membrane fragments at optical density 38 , extracted from the halophilic bacterium Halobacterium halobium were used to determine the thermal energy changes associated with the photochemically-induced intermediates and conformational states of bacteriorhodopsin. Bacteriorho- 


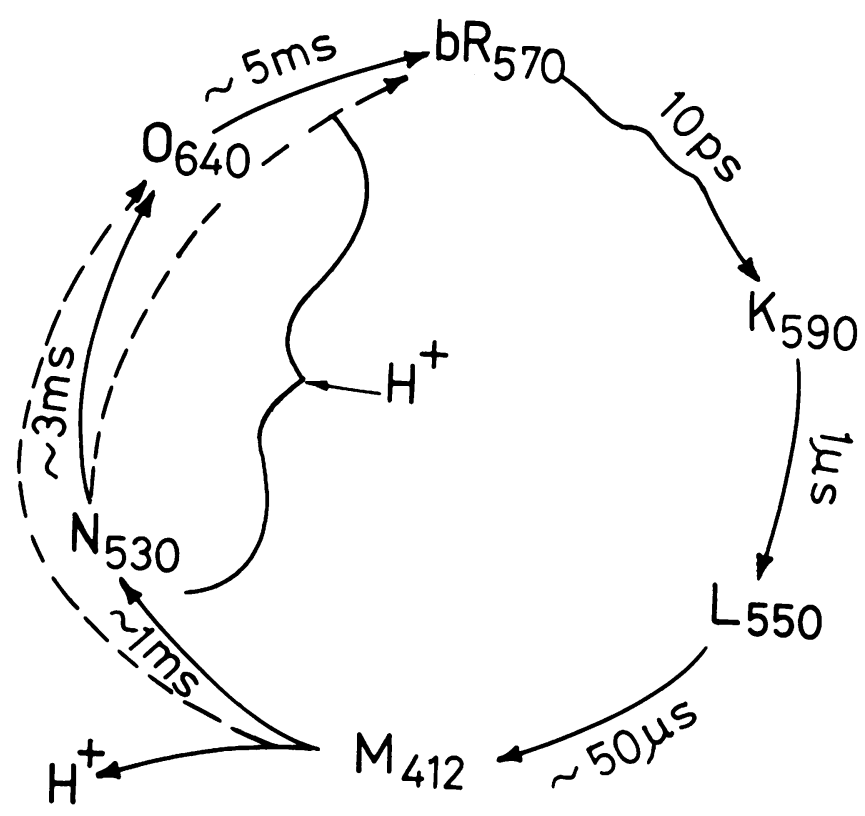

FIGURE 1 The photocycle of bacteriorhodopsin with decay times of the intermediates (taken from reference 1).

dopsin contains a retinal molecule attached to a lysine residue through a protonated Schiff base linkage. A photocycle is initiated by absorption of light by the retinal and involves six known intermediates: $b R_{570}, K_{590}, L_{550}, M_{412}$, $\mathrm{N}_{530}$, and $\mathrm{O}_{660}$ (1). A schematic representation of this photocycle is shown in Fig. 1. Bacteriorhodopsin functions as a light-driven proton pump (1). The protein and associated lipid may undergo conformational changes associated with the mechanism of this pump. We have used photoacoustic measurements to determine the enthalpy changes associated with the photocycle and the conformational changes.

\section{RESULTS AND DISCUSSION}

The photoacoustic technique monitors thermal oscillations in the membrane suspension that are induced by a modulated light input. These thermal changes are sensed as modulated pressure changes by a microphone in contact with the air layer above the sample. The kinetics of the thermal changes are obtained from the frequency $(f)$ dependence of the photoacoustic signal (2). The rate constant of each thermal jump is equal to $2 \pi f$ at the midpoint of the jump. The frequency dependence due to the photoactivity of the protein is superimposed on the frequency dependence due to the decrease in thermal diffusion length as a function of frequency. The photoacoustic data must be normalized by data from a nonphotoactive reference whose frequency dependence results solely from the changing thermal diffusion length and heat transfer across the membrane-aqueous solution boundary. A purple membrane suspension of concentration equal to that of the sample is photobleached with $\mathrm{NH}_{2} \mathrm{OH}(3)$ and

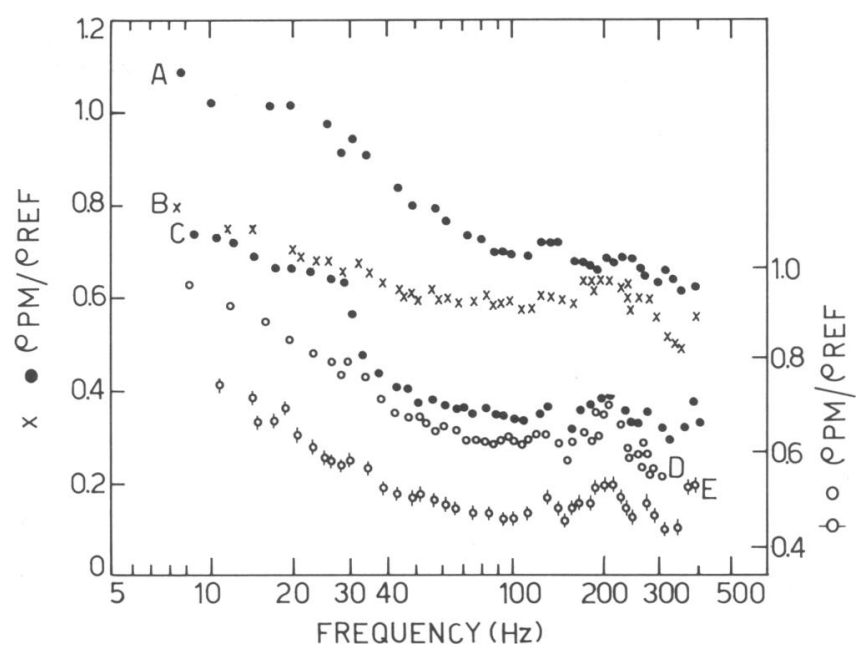

FIGURE 2 The normalized photoacoustic signal as a function of frequency for membrane suspensions at different values of $\mathrm{pH}$ and ionic strength. $P_{\mathrm{pm}} / \boldsymbol{P}_{\text {ref }}$ is the photoacoustic signal of the purple membrane

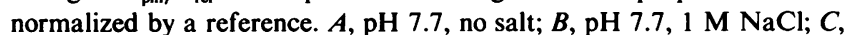
pH 7.0, no salt; $D, \mathrm{pH} 9.0$, no salt; $E$, pH 9.0, $1 \mathrm{M} \mathrm{NaCl}$.

mixed with ink so that the absorption at the measuring wavelength is equal to that of the sample.

Fig. 2 shows normalized frequency spectra of purple membrane suspensions at different values of $\mathrm{pH}$ and ionic strength. The excitation is at $560 \mathrm{~nm}$, near the $570 \mathrm{~nm}$ absorption maximum of bacteriorhodopsin. Exothermic heat jumps are observed in these curves at $35,60,85,130$, 200 , and $300 \mathrm{~Hz}$. The jumps at $130,60 \mathrm{~Hz}$, and $35 \mathrm{~Hz}$ yield rate constants corresponding to the decay times of $\mathrm{M}_{412}, \mathrm{~N}_{520}$, and $\mathrm{O}_{660}$. The jumps at 85,200 , and $300 \mathrm{~Hz}$ may represent energy changes associated with large conformational changes of the protein occurring at $2 \mathrm{~ms}$, $0.8 \mathrm{~ms}$, and $0.5 \mathrm{~ms}$ after illumination. The jump at $35 \mathrm{hz}$ is the most sensitive to changes of $\mathrm{pH}$ and ionic strength. At pH 7.7 and in $1 \mathrm{M} \mathrm{NaCl}$, this jump is smaller than that observed under other conditions. Ort and Parsons (4) and Garty, Cahen and Caplan (2) observed an endothermic change on this time scale. It is possible that several enthalpy changes associated with conformational changes in the protein-lipid complex and with the photoactive pocket of the protein are competing, so that under different conditions the net enthalpy change at $35 \mathrm{~Hz}$ can be endothermic or exothermic. The data in Fig. 2 are preliminary and it is necessary to study the behavior at $\mathbf{3 5}$ $\mathrm{Hz}$ in more detail.

The results suggest that enthalpy changes occur which are associated with changes other than the formation of photocycle intermediates. There may be a net conformational change between $b R_{570}$ and $M_{412}$. The enthalpy changes at $0.5 \mathrm{~ms}, 0.8 \mathrm{~ms}, 2 \mathrm{~ms}$, and $4.5 \mathrm{~ms}$ may reflect the conformational states of the protein-lipid complex as it returns to the original conformation of $b R_{570}$.

Received for publication 7 May 1981. 
1. Stoeckenius, W., R. H. Lozier, and R. Bogomolni. 1979. Bacteriorhodopsin and the purple membrane of Halobacteria. Biochim. Biophys. Acta. 505:215-278.

2. Garty, H. D. Cahen, and S. R. Caplan. 1980. Photoacoustic calorime- try of Halobacterium halobium photocycle. Biochem. Biophys. Res. Commun. 97:200-206.

3. Oesterhelt, D., and W. Stoeckenius. 1971. Rhodopsin-like protein from the purple membrane of Halobacterium halobium. Nature New Biol. 233:249-152.

4. Ort, D. R., and W. W. Parsons. 1979. Enthalpy changes during the photochemical cycle of bacteriorhodopsin. Biophys. J. 25:355-364.

\title{
THE RELATIVE LOCATIONS OF INTRAMEMBRANE FLUORESCENT PROBES AND OF THE CYTOSOL
}

\section{HEMOGLOBIN IN ERYTHROCYTES, STUDIED BY}

\section{TRANSVERSE RESONANCE ENERGY TRANSFER}

\author{
J. EISINGER AND J. Flores \\ Bell Laboratories, Murray Hill, New Jersey 07974 U.S.A.
}

We have investigated the use of transverse resonance energy transfer (RET) to determine the level of fluorescent probes within the phospholipid regions of the erythrocyte membrane relative to the plane of closest approach of the cytosol hemoglobin. The $n$-(9-anthroyloxy)-stearic and palmitic acids ( $n$-AS and $n$-AP) and 9-vinyl anthracene (9-VA) embedded in the membrane were used as donors, and the hemes of the cytosol hemoglobin were the acceptors.

The experiments were analyzed by a model in which the acceptor hemes are constrained to remain in the half space defined by a plane whose normal distance from a donor $(D)$ is $d$. The hemes are assumed to be uniformly and continuously distributed and their orientations to be dynamically and isotropically averaged. It can be shown that at the high heme concentration inside the erythrocyte $(20 \mathrm{mM})$ and the modest efficiencies of RET encountered here, this model is adequate for determining at least an approximate value of $d$, but in any case a $d_{\max }$.

\section{RESULTS}

Following Wolber and Hudson (1), it is convenient to express the distances and the acceptor density $(\rho)$ as dimensionless parameters, so that $x=d / R_{0}$ and $\rho$ is the number of acceptors per volume $R_{0}^{3}$, where $R_{0}$ is the usual Förster distance. The desired equation for $x$ may then be obtained analytically in terms of the measured average RET efficiency, $<T>$

$$
d=x R_{0}=\left[(\pi \rho / 6)\left(<T>^{-1}-1\right)\right]^{1 / 3} .
$$

This relationship is illustrated graphically in Fig. 1.

$<T>$ was determined by measuring the fluorescence decay of the membrane-bound probes in intact erythrocytes and ghosts made from labeled cells. The decays were measured by means of a monophoton lifetime instrument using a high-pressure hydrogen spark lamp of 2.5-ns half-width. The experimental time profiles were fitted by multi-exponential decay functions of the type

$$
I(t)=\sum \alpha_{\mathrm{i}} \exp \left(-t / \tau_{\mathrm{i}}\right)
$$

after convolution by the excitation pulse profile. One may then define the mean lifetime of the donor by

$$
<\tau>=\sum \alpha_{\mathrm{i}} \tau_{\mathrm{i}}^{2},
$$

since $\alpha_{\mathrm{i}} \tau_{\mathrm{i}}$ is the fraction of light with life time $\tau_{\mathrm{i}}$. By comparing $<\tau>$ for intact cells and ghosts (subscripts $\mathrm{c}$ and $\mathrm{g}$ ), i.e., in the presence and absence of the heme acceptors,

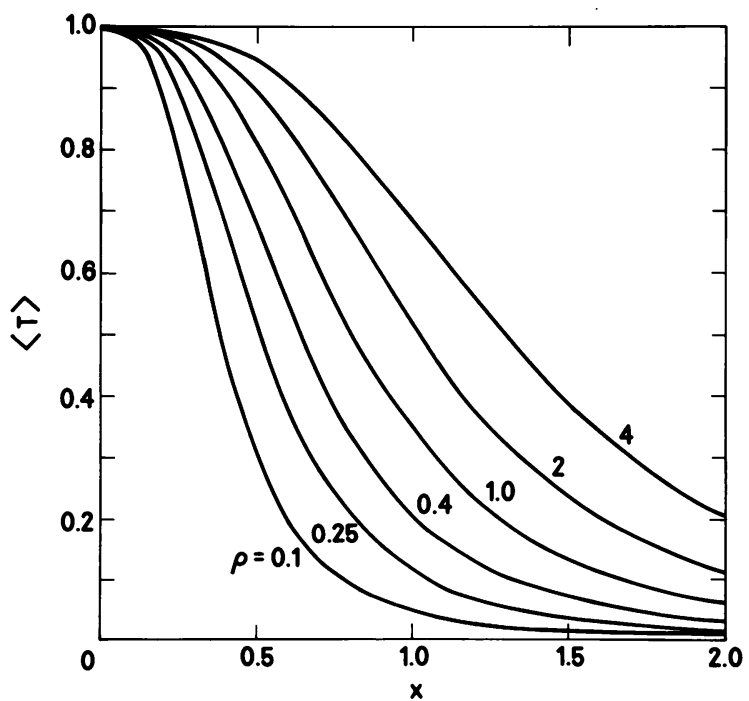

FIGURE 1 The dependence of $\langle T\rangle$, the dynamically averaged RET efficiency from a donor, $D$, to a continuum of acceptors beyond a plane whose normal distance from $D$ is $x, x$ is a dimensionless quantity measured as a multiple of the Förster distance $R_{0} . \rho$ is the density of acceptors per volume $R_{0}^{3}$. 